\title{
DISTRIBUTED SELF-SIMULATION OF HOLONIC MANUFACTURING SYSTEMS
}

\author{
Naoki Imasaki ${ }^{1}$, Ambalavanar Tharumarajah $^{2}$, Shinsuke Tamura ${ }^{3}$ \\ ${ }^{1}$ Toshiba Corporation, Japan, naoki.imasaki@toshiba.co.jp \\ ${ }^{2}$ CSIRO Manufacturing Science \& Technology, Australia, rajah.tharumarajah@csiro.au \\ ${ }^{3}$ Fukui University, Japan, tamura@fuis.fuis.fukui-u.ac.jp
}

\begin{abstract}
This paper details the development of a distributed simulation capability for holonic manufacturing systems based on the concept of self-simulation. Every holon in the system would function similar to an autonomous simulator that maintains its own clock and event execution. This paper discusses synchronization and other technical issues in designing such a simulator and puts forward the basic requirements and design options for its implementation. These are results of HMS project in IMS (Intelligent Manufacturing Systems) program.
\end{abstract}

\section{INTRODUCTION}

Simulation is an effective tool for examining how a system works. For Holonic Manufacturing Systems (HMSs; Christensen, 1994), simulation becomes critical because HMSs are often too large-scale and distributed to handle their behavior analytically. Further, holons in HMSs exhibit non-deterministic behaviors and deal with discrete events like human operations, thus the simulation should be of discrete-event naturally.

Simulation of HMSs fulfills four core capabilities that are critical for designing and operating HMSs. First, it is used to test the functional and logic correctness of a proposed system during design. Second, it is used in analyzing faults during operation. Third, it is used for implementing intelligence in the holons. This enables a holon to assess future performance and decide on a course of actions. Lastly it is used to test task execution performances, e.g. response time and throughput.

To provide the above capabilities, a simulator of HMSs should ideally be modifiable and extendable as and when changes in design or operation of the system takes place. Also, it should permit easy switching between normal (i.e. actual operation) and simulation modes of operation of a holon. A distributed selfsimulator is proposed to satisfy these features. The idea behind a distributed selfsimulator is firstly to provide holons with their own simulation capabilities (their operation mode can be changed by their mode switch, i.e., "normal" and "simulation"), and secondly to create a simulation capability as a set of independently acting self-simulators having their own processes and that can be remotely connected over a network. A single self-simulator (hereafter called a 
logical process or LP (Fujimoto, 2000)) would process events in strict time sequence, generate new events and communicate with other LPs. In a holonic system, an LP can be representative of a holon or a sub-set of holons. Compared to the traditional centralized approach of using a single sequential simulator for the entire system, this approach has the following advantages: system simplicity, modularity, extensibility, maintainability, and high computation performance.

This paper is structured as follows. Section two describes the architecture and the high level functional requirements of the proposed distributed simulator. Section three elaborates the requirements for the development of the distributed simulator. Next features a prototype is briefly described followed by conclusion

\section{GENERAL REQUIREMENTS}

\subsection{Architecture}

The architecture of the distributed self-simulator is shown in Figure 1.

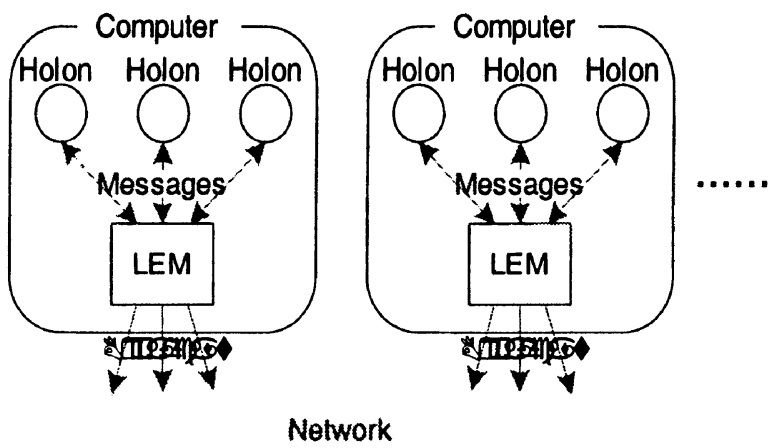

Figure 1 - Communication Architecture

In this architecture, a set of holons is defined to operate under a single Local Event Manager (LEM). Likewise, another set of holons can be defined to operate under a different LEM. This architecture and the development are based on JDPS (Seki, 1991), a Java based HMS execution platform. A member holon wishing to send a message would forward it to an LEM to which the holon belongs. The LEM would broadcast the message to other LEMs and also check whether the receiver holon is one of the members. If it were the latter, the message would be internally forwarded to that holon. On receiving a message from another LEM, an LEM would check whether the receiver holon is a member and if so would forward it to the holon. If this is not the case, the message will be ignored.

This architecture is used to define and construct the holons of the distributed simulator. Two distinct configurations are possible:

LEM as an LP (LEM-LP): An LEM acts as a single simulator. It manages the ordering and execution of incoming events and maintains its own local clock. The holons would receive the events, process them and any new events generated would be forwarded to the LEM. Inconsistencies among events are detected by the LEM and appropriate actions are taken to correct such inconsistencies.

Holon as an LP (Holon-LP): A holon acts as a single simulator and, hence, manages the ordering, execution and generation of events. Each holon 
maintains its own clock and any inconsistencies among events are detected and rectified by the holon. LEM acts purely as a message router.

The simulator that this paper proposes is the mixture of LEM-LP and Holon-LP configurations, i.e., local clocks are managed by LEMs and event inconsistency is detected and corrected by holons.

\subsection{Functional Requirements}

An overriding requirement on the performance of a distributed simulator is that it must produce the same result as that of a single sequential simulator for the same data. This requires the LPs (i.e. distributed simulators) to correctly synchronize their local clocks with each other as well as to maintain perfect consistency of states. However, a distributed simulator is markedly different in its architecture and its performance depends on the adequacy of the underlying communication network and memory. The following sub-sections first describe the assumptions followed by the requirements on synchronization.

\subsubsection{Assumptions}

The following assumptions are made with respect to the underlying communication network and the computational environment of LPs (modified and extended from $p$. 98, Fujimoto, 2000):

Assumption 1: There are no state variables shared between LPs: This is to say that LPs manage their state dependencies primarily through communication.

Assumption 2: Communication among the LPs is reliable: That is, every message that is sent eventually arrives at the receiver.

Assumption 3: An LP sends messages in its local-timestamp order.

Assumption 4: A communication network guarantees that messages are delivered in the same order as they were sent.

Assumption 5: LPs may be destroyed or new LPs created during the execution of simulation.

Assumption 6: Memory at each LP for storing states is limited: Memory overflow when storing states can cause fatal errors and should be avoided.

\subsubsection{Requirement of Synchronization}

In the distributed simulation environment an LP manages the ordering and execution of local events and maintains the local simulation clock. There are two kinds of events in the simulator, one is a pure-simulation event that signifies the start or finish of an activity, and the other is an event corresponding to a message delivered. The types of activities can be associated with the LPs (or holons representing physical devices) and include processing activities with time delays and other message sending/receiving actions. An event would have a timestamp (local clock time) for its occurrence and a recipient for its execution.

An LP chronologically orders the events according to time of occurrence (i.e., the timestamp), and posts the first event for execution. The time in the local clock is 
incremented to the timestamp of the event that is being executed. This way, the time within an LP is maintained (i.e. local synchronization)

However, along with the local synchronization, a mechanism should exist to assure synchronization of events among the LPs. The problem of nonsynchronization occurs due to the independent adjustments of local clock times by the LPs. To elaborate this problem, consider a message sent to $\mathrm{LP}_{\mathrm{A}}$ at time $t_{3}$ from another LP as shown in Figure 2. $\mathrm{LP}_{\mathrm{A}}$ finds that the event \#3 (hereafter referred to as a straggler event) is in the past (current time at $\mathrm{LP}_{\mathrm{A}}$ is $t_{A}>t_{3}$ ). This means that there is a difference of $t_{A}-t_{3}$ between the clock time of the LP that sent the message and the local clock time. An immediate consequence of the straggler event is that the state of $\mathrm{LP}_{\mathrm{A}}$ in this time period is invalid or has to be re-validated. The consistency among the simulated states of $\mathrm{LP}_{\mathrm{A}}$ can be assured only up to time $t_{3}$.

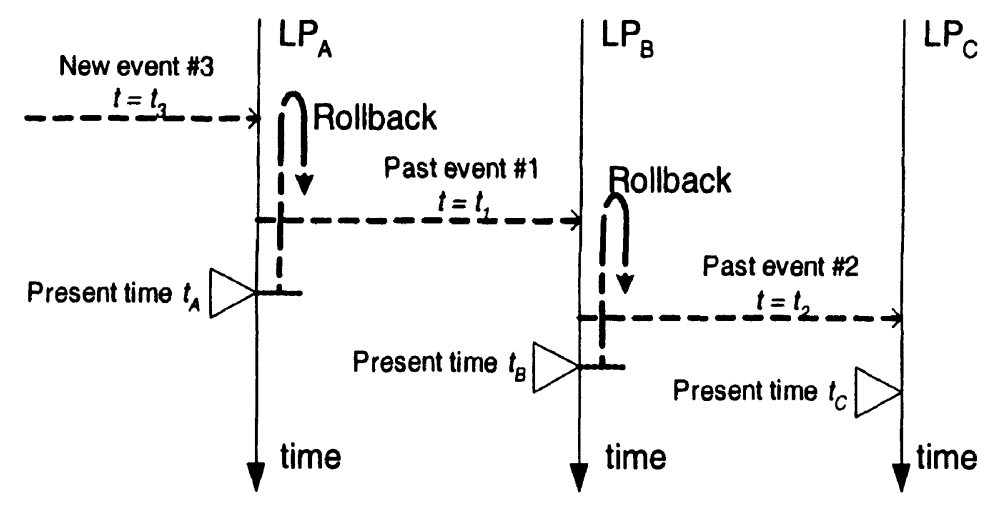

Figure 2 - Event synchronization and rollbacks

To remove the inconsistency, $\mathrm{LP}_{\mathrm{A}}$ should ideally rollback its state to that which prevailed at time $t_{3}$ and re-execute the events (including the one received) that occurred in the intervening period $t_{A}-t_{3}$. The rollback would cancel all the events that the holon posted with a timestamp later than $t_{3}$, execute the straggler event and then re-execute the simulation from $t_{3}$. The rollback in a one holon, however, can lead to retraction of events that were previously scheduled for other holons and a consequent propagation of straggler events. For instance, the retraction of event \#1 posted from $L P_{A}$ to $L P_{B}$ at time $t_{1}>t_{3}$ would cause $\mathrm{LP}_{\mathrm{B}}$ to rollback. This, in turn, would cause a retraction of the event posted by $\mathrm{LP}_{\mathrm{B}}$ to $\mathrm{LP}_{\mathrm{C}}$ at time $t_{2}$ and so on. The local detection and correction of inconsistencies using rollback, thus, provides a mechanism for ensuring the states of the LPs are globally consistent with each other.

In order to satisfy the requirements of synchronization and to perform adequately under the above assumptions, a simulator needs the following essential functions:

1) Detection of inconsistencies

2) Rollback of events to maintain a consistent state within and across LPs

3) State saving that conserves the memory

4) Recursive rollback avoidance (RRA)

These functions are elaborated in the next section. 


\section{REQUIREMENTS SPECIFICATION}

This section elaborates the functions required to construct the distributed simulator and suitable strategies for their implementation.

\subsection{Detection of Inconsistencies}

As observed before, a distributed simulator should be capable of producing the same final result as a sequential simulator. This could be guaranteed by ensuring that each holon satisfies the local causality constraint, i.e., when each holon is able to process the events in timestamp order and thereby ensures the correct mapping of the state space of a holon. However, in a distributed implementation of LPs, there can be occurrence of straggler events, thus violating the local causality constraints.

When an LP receives a straggler message it has to first detect the inconsistency and correct it by undoing the inconsistent events, i.e., through a rollback. The possible ways to detect inconsistencies are as follows.

Time inversion inconsistency detection (TIID): If the timestamp $t_{S}$ of a received event satisfies $t_{S}<t_{C}$ where $t_{C}$ is the local simulation time of the $\mathrm{LP}$, it is unconditionally judged as an inconsistency occurrence.

Semi-semantic inconsistency detection (SSID): If the timestamp $t_{S}$ of a received event satisfies $t_{S}<t_{C}$ where $t_{C}$ is the local simulation time of the LP and the action invoked by the event changes the state of the LP, it is regarded as an inconsistency occurrence. However, if there are no events that fetch the state during $t_{S}<t \leq t_{C}$, then there is no inconsistency.

Obviously SSID can reduce redundant rollbacks though it may require additional computation.

Because rollback can be computationally intensive, inconsistency is detected at the level of a holon, instead at the level of an LEM as in conventional distributed simulators. Holon-wise inconsistency checking connected with LEM-wise event ordering reduces the frequency of inconsistency. Namely time inversion in an LEM does not always imply time inversion in individual holons. On the other hand, an LEM insures that events posted by holons within itself are consistent.

\subsection{Rollback}

Retraction of events due to a rollback can employ one of two methods, viz. aggressive and lazy cancellation (Fujimoto, 2000). Aggressive cancellation is when an event $E$ that is being retracted by an LP sends a cancel message to retract all events that the LP posted after $E$. In lazy cancellation, no such message is sent until the rolled back event is recreated and the validity of the previously scheduled event is examined. Thus, lazy cancellation offers the advantage of avoiding retracting events that are recreated when rolled back events are reprocessed, though more computation is required. The semi-semantic inconsistency checking can be directly implemented for lazy cancellation.

It should be noted that if the assumption on correct network delivery is violated (i.e., the network cannot guarantee the delivery of messages in the same order as 
they are sent), then an additional cancellation of all processed events with a timestamp equal to that of the straggler event becomes necessary.

A holon can initiate rollback a) locally, b) LEM-wide or c) system-wide. Types b) and c) help to re-synchronize clock times of a sub-set of holons. However, a similar effect can be obtained with local rollback through propagation of event cancellation with less computation.

Holon-wise (local) rollback: When a holon rollbacks to time $t_{s}$, it sends rollback requests only holons that received events from it.

LEM-wide rollback: When a holon belonging to an LEM rollbacks to time $t_{s}$, it sends rollback requests to all holons belonging to the LEM to rollback to time $t_{s}$.

System-wide rollback: When a holon rollbacks to time $t_{s}$, it sends rollback requests to all holons in the system to rollback to time $t_{s}$.

A rollback operation is when a holon, detecting inconsistency, re-initializes its state at the current clock time $t_{c}=t_{c}^{\prime}$ to a previous state (at time $t_{s}$ ) and then proceeds to re-construct its state-space up to the current clock time. The followings show the comparison between the aggressive cancellation and the lazy cancellation.

Aggressive cancellation (with local rollback):

Step 1. A straggler event $E_{j}\left(t_{s}\right)$ received by holon $H_{j}$ causes rollback to $t_{s}$

Step 2. $H_{j}$ sends cancellation events $E_{k}^{c}(t)$ to holons $H_{k}$ of previously scheduled events $E_{k}(t)$ where $t_{s}<t \leq t_{c}^{\prime}$

Step 3. $H_{j}$ executes straggler event $E_{j}\left(t_{s}\right)$ and resets the local clock time $t_{c}=t_{s}$

Step 4. $H_{j}$ executes an RRA (discussed in Section 3.4) procedure

Step 5. $H_{j}$ re-executes a previously received event or executes a newly received events (includes a resent event) $E_{j}\left(t_{0}\right)$ where $t_{0} \leq t_{c}^{\prime}$ in ascending order of time and resets the local clock time to $t_{c}=t_{0}$

Step 6. $H_{j}$ schedules new events $E_{k}\left(t_{0}\right)$, if appropriate, to holon $H_{k}$

Step 7. Goto Step 5

Lazy cancellation (with local rollback):

Step 1. A straggler event $E_{j}\left(t_{s}\right)$ received by holon $H_{j}$ causes rollback to $t_{s}$

Step 2. $H_{j}$ executes straggler event $E_{j}\left(t_{s}\right)$ and resets the local clock time $t_{c}=t_{s}$

Step 3. $H_{j}$ re-executes a previously received event or executes a newly received events $E_{j}\left(t_{0}\right)$ where $t_{0} \leq t_{c}^{\prime}$ in ascending order of time and resets the local clock time to $t_{c}=t_{0}$

Step 4. $H_{j}$ re-validates a previously scheduled event $E_{k}(t)$ where $t_{s}<t \leq t_{c}^{\prime}$ and if $E_{k}(t)$ is not valid, $H_{j}$ sends a cancellation event $E_{k}^{c}(t)$ to holon $H_{k}$ 
Step 5. $H_{j}$ schedules new events $E_{k}\left(t_{0}\right)$, if appropriate, to holon $H_{k}$

Step 6. $H_{j}$ executes an RRA procedure

Step 7. Goto Step 3

\subsection{State Saving}

When a straggler event occurs and events are cancelled, an LP should ensure that a consistent state-space mapping prevails. This is achieved by rolling back the state variables in time. Thus, rollback operation requires storing the states at each state change or event execution (called checkpoint saving) in order that the desired state at some point in time in the past can be restored.

Checkpoint saving at each event consumes more and more memory as new events are created, but it seldom releases memory. This situation is not acceptable due to the finite availability of memory (assumption 6) at an LP. Therefore, a mechanism should be constructed to limit the storing of state variables over the simulated period. While infrequent saving of state can reduce memory needs, it too cannot be used to reclaim memory.

This problem can be overcome by determining the events that cannot be rolled back and hence the time before which no checkpoint saving is necessary. Computing the lower bound on the timestamp among all unprocessed and partially processed messages in the system referred to as Global Virtual Time (GVT) could identify these events. Thus, a requirement for an efficient checkpoint saving is the computation of GVT using an appropriate algorithm, though it should be pointed out that this may or may not be implemented in preference to simpler local methods.

\subsection{Recursive Rollback Avoidance}

In (re) scheduling an event after a local rollback, a holon can face the following situation. If a cancelled event is found to be valid, the holon will schedule a new event. However, due to the previous cancellation, the holon can expect to receive a response event. It is possible that the timestamp of the response event happens to be smaller than the local clock time. Such a case would require another rollback during a rollback procedure. This is computationally quite ineffective.

The following are some of mechanisms that can be explored to avoid or minimize recursive rollbacks.

Local Fixed Wait: A simple mechanism to avoid a recursive rollback is for the holon to wait (in real-time), after it re-executes scheduled events.

Controlled event execution: A holon controls the speed of re-execution after rollback, thus, permitting other holons to respond.

Conservative execution: Holons involved in the rollback adopt conservative event handling strategy to eliminate time inversion during rollback operations. In this case, events that are received before the rollback can be used as look-ahead.

To select the best method that guarantees the synchronization of rollback is one of the most important topics. Local fixed wait method is simplest, though investigations are required to determine its performance. 


\subsection{Design options}

The following summarizes the design options that are selected to minimize communication and computational loads and to achieve adequate performance:

$$
\text { Configuration: LEM-LP }
$$

Detection of inconsistencies: Holon-wise SSID

Rollback: Lazy cancellation

State saving: Event-wise saving with GVT estimation

Recursive rollback avoidance: Local fixed wait

However, further investigations will be carried out to ascertain the sufficiency of performance, and if necessary other appropriate options may be investigated.

\section{PROTOTYPING}

A prototype of the distributed self-simulator, called simJDPS is developed based on the architecture we discussed above. Each holon is endowed with simulation capabilities to maintain their respective local clocks and event lists. Communication is indirect and is mediated by a Site Manager, acting as an LEM. In essence, the holons function similar to their actual counterparts, except for the passage of time that is simulated rather than real.

simJDPS is implemented on JDPS by extending existing classes and by adding new classes. For example, simHolon class extends the Holon class of JDPS to implement the self-simulation capability in holons, and SiteManager class is modified to act as LEM. The first prototype model incorporates Holon-LP configuration, TIID, aggressive cancellation with local rollback and event-wise check point saving. This model was tested for its functionality and run for a 2-site multi-holon situation.

This model will be extended to include mechanisms for lazy cancellation, computation of GVT and RRA.

\section{CONCLUSION}

Distributed self-simulation of holons in a HMS has many advantages, both during design and operation. However, there are many technical challenges in developing such a simulator including ensuring perfect synchronization, minimizing rollback situations, preventing memory overflows and above all maintaining a performance that at least matches that of a single centralized simulator. The model and the specifications detailed in this paper attempts to address these challenges by proposing a novel distributed self-simulation approach.

\section{REFERENCES}

1. Christensen, J. Holonic Manufacturing Systems - Initial Architecture and Standards Directions, 1st Euro. Conf. on HMS, Hanover, Germany, 1994.

2. Fujimoto, RM. Parallel and Distributed Simulation Systems, John Wiley \& Sons, Inc., 2000.

3. Seki, T, Hasegawa, T, Okataku, Y, Tamura, S. An Operating System for Intellectual Distributed Processing System - An Object Oriented Approach based on Broadcast Communication -. J. of Information Processing, Vol. 14, No. 4, pp.405-413, 1991. EMBED 